\title{
Hearing Problems in Children and Mowat-Wilson Syndrome: A Case Report
}

\author{
Ben Ahmed Wided* \\ ENT Specialist, Private Clinic, Tunisia
}

*Corresponding author: Ben Ahmed Wided, ENT Specialist, Private Clinic, Former resident of Tunis Hospitals, Tunisia

Keywords: Deafness; dysmorphia; epilepsy; molecular study; MowatWilson syndrome

Abbreviations: MSW:Mowat-Wilson syndrome; AEP: Hearing Potentials

\section{Introduction}

Hearing problems, more precisely hearing loss in children, is a very frequent reason for consultation these days. In early childhood, these disorders affect language and communication, and deafness is the most common sensory deficit. According to the WHO, the hearing-impaired child is one whose hearing acuity is insufficient to enable him to learn his own language, to participate in the normal activities of his age and to follow with profit general school education [1]. Its prevalence is estimated between 1 and 3.4 per thousand at birth [2] and could reach 1 per 100 in cases of severe perinatal disease and the genetic origin is then implicated in $60-80 \%$ of cases [3]. But the etiologies differ, and hypoacusis can even be a symptom of congenital syndromic disease.

\section{Case Study}

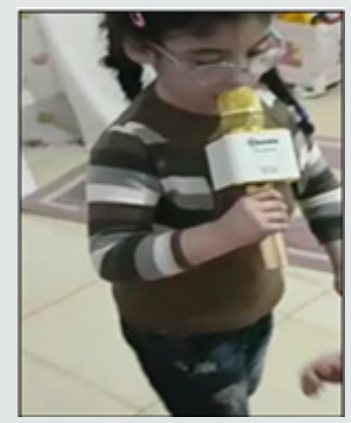

Figure 1: Hypertelorism; large eyebrows; prominent columella.
In our work we report the case of a 3-year-old girl from Algeria brought by her parents to Tunisia to take charge of hearing loss, according to them; presenting a language delay that has progressed for 2 years; with a particular dysmorphic facies and unexplored convulsive seizures. The interrogation clarified an outconsanguineous marriage; normal course of pregnancy with an uneventful vaginal delivery (according to the health record) with a birth weight of $3,400 \mathrm{~kg}$. moreover, the child's vaccination schedule was respected. The history of the disease dates back to the age of 18 months when the parents noticed delayed speech as well as hearing loss on call with the onset of seizures. , The clinical examination beginning with the inspection showed marked hypertelorism especially in the upper lip; high forehead with two frontal bumps; large eyebrows poor in their middle, saddle nose and prominent columella (Figure 1); large raised lobes with a central depression (Figure 2); blue staining of the eardrums on otoscopy with congestive appearance of the nasal mucosa and hypertrophy of the inferior turbinate's. Nasofibroscopy performed eliminated the presence of adenoids. The rest of the examination is normal with a weight of $18.4 \mathrm{~kg}$ and a blood pressure of 100/60 mmHg. Additional audiometric examinations have revealed bilateral serummucous otitis with a flat line on the tympanometry and a normal threshold at PEA and deafness transmission of 30 DB to behavioral audiometry. A specific assay of specific pediatric pneumallergenic IgE revealed a large number of allergens in children (Figure 3). A medical treatment based on oral corticosteroid therapy at a dose 
of $2 \mathrm{mg} / \mathrm{kg}$ associated with antibiotic therapy (clavulanic acid12.5 $\mathrm{mg}+$ amoxicill100 $\mathrm{mg}$ ) at a dose of $80 \mathrm{mg} / \mathrm{kg}$ and local nasal treatment based on corticosteroid therapy and nasal washings with sprays based on essential oils. In the meantime, we have further explored within the framework of the exploration of epilepsy and the particular dysmorphic facies.

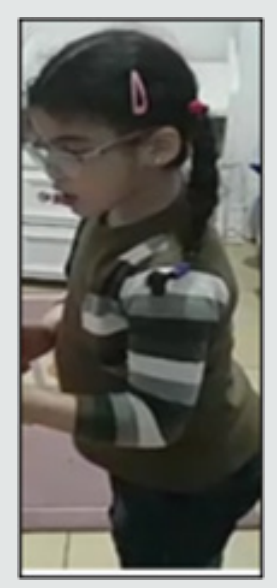

Figure 2: Large earlobes.

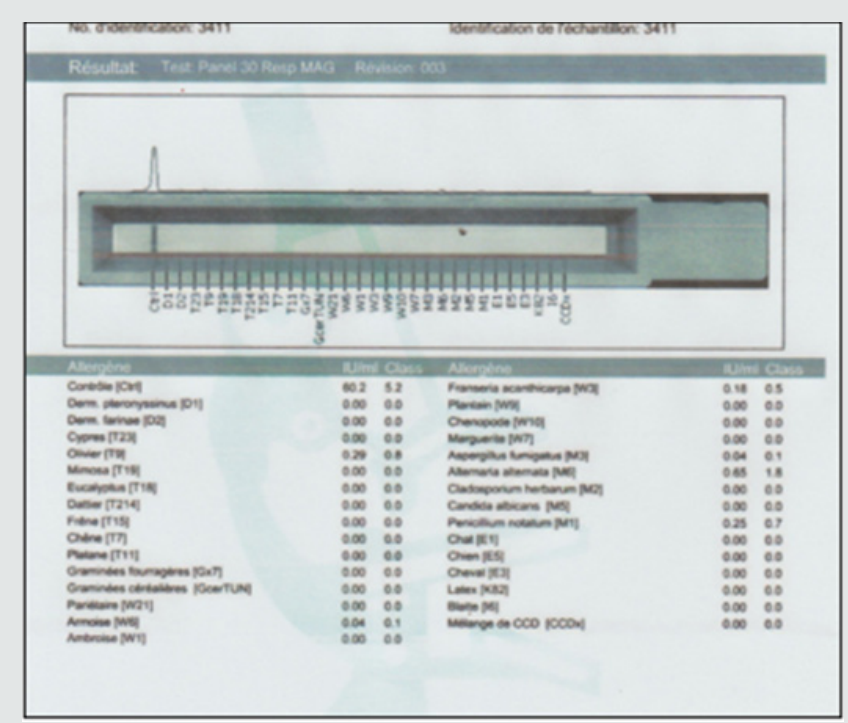

Figure 3: Dosage of specific pneum allergenic IgE positive.

An EEG performed showed a diffuse slowing of the background rhythm with brief generalized spikes. MRI of the brain and ponto-cerebellar angles returned strictly normal especially no microcephaly or agenesis of the corpus callosum and an intact inner ear. Before epilepsy and dysmorphic facies, a congenital syndrome was suspected and the first that came to mind was Dravet's syndrome, given the generalized epileptic seizures, hence a genetic study (total sequencing of the exome) of the child was performed revealing the diagnosis of Mowat-Wilson syndrome (Figure 4). A second genetic study of the parents' genome was made and returned without suspicious abnormalities. The now multidisciplinary care (neuropediatrician, speech therapist and occupational therapist) has led to good further development; sedation of convulsive seizures under an anti-epileptic drug at a dose of $500 \mathrm{mg} /$ day and language acquisition around the age of 3 years 6 months and resumption of an active life with the children (patient put in daycare; follows swimming at the pool).

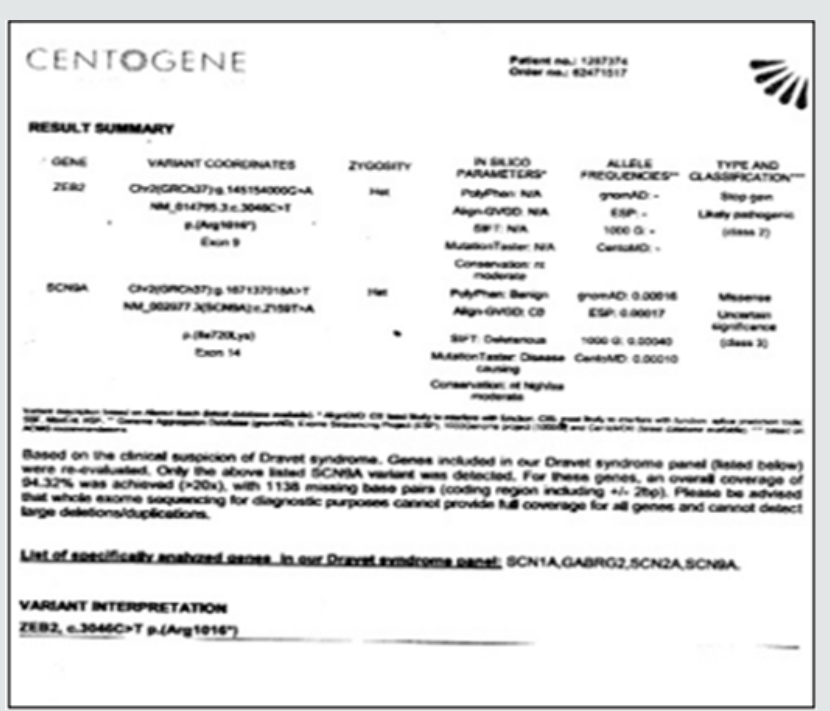

Figure 4: Sequencing of the exome.

\section{Discussion}

Childhood deafness is a very common health problem, usually associated with delayed or impaired speech. All origins combined; deafness affects 1.3 to 2.3 children out of 1000 in industrialized countries. Deafness can be classified into 2 categories according to the mechanism $[2,4]$. Conductive hearing loss linked to damage to the outer ear or the middle ear. They are acquired in $99 \%$ of cases and are most often accessible to medical treatment +/- surgical. Their etiology is dominated in young children by inflammatory and infectious pathologies linked to tubal dysfunction as it is in our case. They can also be secondary to malformative, traumatic or tumor pathologies of the pinna, the external auditory canal or the ossicles. This deafnessis most common in children and do not exceed $60 \mathrm{~dB}$ of hearing loss; $95 \%$ of this deafness are linked to otitis media. Congenital conductive hearing loss accounts for $0.5 \%$ of conductive hearing loss.

Major aplasia: The frequency of debilitating bilateral forms varies greatly depending on the series, probably in the order of 20 to $30 \%$. Four grades have been described from turbinate ear (grade I) to anotia (grade IV) resulting in conductive hearing loss of 60 to $70 \mathrm{~dB}$. Minor aplasia: the malformation of the middle ear is isolated or associated with minor malformations of the outer ear or certain polymalformative syndromes (in our case large lobe with a well calibrated external auditory canal). 
Sensorineural hearing loss which may be secondary to pathology of the organ of Corti, the auditory nerve and / or central auditory areas [5]. Hearing loss is extensive from mild to complete and is very commonly associated with message distortions. This deafnessis in two thirds of the cases genetic, autosomal recessive in $80 \%$ of the cases, autosomal dominant in $20 \%$ of the cases, linked to the $\mathrm{X}$ affecting only rare boys, or mitochondrial (deaf mother transmitting to all her children ) exceptional. The consequences of this high prevalence make it possible to carry out a routine molecular diagnosis and to confirm the genetic nature of deafness, thus giving the parents the risk of recurrence $(25 \%)$ for future births. Whatever the mode of transmission, sensorineural hearing loss is either isolated (two thirds of cases) or associated with a polymalformative syndrome. Radiological exploration (MRI) associated with a study of hearing potentials (AEP) associated with a genetic molecular study confirms the diagnosis. In our case, the cochlear and genetic origin was eliminated (normal inner ear and PEA). Mixed deafness which results from damage to the outer and / or middle and inner ear.

Deafness can be classified according to the date of onset $[4,6]$

a) The audio-phonatory loop is necessary for the development of the child's language and speech, its interruption therefore does not have the same consequences depending on the age of onset of deafness.

b) Congenital deafness: deafness exists from birth.

c) Pre-lingual deafness (where our case is; hearing loss since the age of 18 months): occurring before the age of 2 years, are the most serious because the early acquisitions essential for subsequent language development do not take place.

d) Perilingual deafness:Occurring between 2 and 4 years

e) Postlingual deafness:Itoccurs after the age of 4 years.

The careful etiological assessment is therefore an essential step for many reasons: genetic counseling only becomes possible with a precise diagnosis, especially in the face of deafness associated with a polymalformative syndrome and the progressive prognosis and the management will subsequently depend on the cause ; as in our case we followed the research protocol for a congenital syndromic etiology which led us, to everyone's surprise, to a rare syndrome still under study: Mowat-Wilson syndrome (MWS) which is a syndrome Multiple birth defects characterized by a distinct facial phenotype, intellectual disability, epilepsy, Hirschsprung's disease (HSC), and variable birth defects. The prevalence is estimated between 1 / 50,000 and 1 / 70,000 live births [7]. More than 200 patients have been reported to date. It seems likely that MWS is underdiagnosed, especially in patients without HSC.The typical characteristic facies of MWS includes a high forehead, frontal boss, large eyebrows flared medially and sparse in the middle part, hypertelorism, deep but large eyes, wide and raised earlobes with a central depression, a saddle nose with a prominent rounded nasal end, prominent columella, open mouth with M-shaped upper lip and prominent but narrow and triangular pointed chin (same description for our patient). The facies become more pronounced with age. The associated HSCR causes constipation which frequently persists after surgery. Patients generally have moderate to severe intellectual disability.

Speech is absent or limited to a few words, with an onset around 5-6 years old. Seizures are common; all types of seizures (absence, generalized tonic-clonic seizures, myoclonic and focal seizures) have been reported. Most of the subjects have a cheerful demeanor with frequent smiles and a sociable personality. Other associated congenital anomalies include cerebral anomalies (agenesis of the corpus callosum), heart (persistence of the ductus arteriosus, interventricular communication,pulmonary stenosis, valve), pulmonary (arterial sling, with or without tracheal stenosis), genitourinary (hypospadias, cryptorchidism, vesicoureteral reflux and hydronephrosis), ocular (microphthalmia) [in our case high myopia] and musculoskeletal (flatfoot, calcaneovalgus) This syndrome is caused by heterozygous mutations or deletions in the zinc finger E box binding gene for homeobox 2, ZEB2, (2q22.3) previously called ZFHX1B (SIP1) [8,9]. To date, over 100 deletions/ mutations have been reported in patients with a typical phenotype; these are often whole gene deletions or truncated mutations, suggesting that haploinsufficiency is the main pathological mechanism. Genotype-phenotype analysis studies show that facial gestalt and retarded psychomotor development are constant clinical features, while frequent and severe birth defects are variable. In a small number of patients, unusual mutations can lead to an atypical phenotype. The clinical diagnosis is then based by summarizing according to Zweier et al. [10] on the distinct facies and the ZEB2 mutational analysis.

Congenital malformations and seizures require early clinical investigation with the intervention of several specialists (including neonatologists and pediatricians) if the diagnosis is suspected early. In addition, prenatal diagnosis has become available and essential for subsequent pregnancies of parents with an affected child.The majority of cases of MWS reported so far were sporadic as in our case, however, germline mosaicism has been described. and the risk of recurrence was calculated at 2\% [11] (his younger brother is strictly normal) Psychomotor development is delayed in all patients (ditto for our case), which is why rehabilitation (physiotherapy, psychomotricity and speech therapy ) should be started as soon as possible (for us, we started it as soon as the diagnosis made at the age of 3 years 2 months. Mortality and morbidity depend on elsewhere about the presence and severity of birth defects. It has been reported according to the literature that patients live in early adulthood but need help with activities of daily living. 


\section{Conclusion}

Hearing is an infinitely precious sense for a child. It allows not only to perceive the outside world, but also to access information and communication. Any impairment of hearing function constitutes a handicap with significant socio-professional repercussions.Any suspicion of hypoacusis in the child must lead to an otolaryngological (ENT) examination and audiometric examinations appropriate to his age to rule out or confirm the hearing loss and, in the latter case, specify the mechanism and the degree and further exploration if there are any associated dysmorphic signs while keeping in mind the variability of congenital syndromes as it was in our case; knowing that Mowat-Wilson syndrome (SMW) is a complex congenital syndrome Described in 1998 by Mowat et al. with a prevalence still unknown and under study.

\section{References}

1. Roussey M Hearing Review.

2. Pol C (2003) Epidemiology and etiologies of deafness in children. Arch Pediatr 10(1): 148-163.

3. Vincent C (2011) Genetic deafness: update. The Journal of Internal Medicine 32S: S2-S4.
4. Mondain M, Blanchet C, Venail F, Vieu A (2005) Classification and treatment of deafness in children. Encycl Med chir Otorhinolaryngology 2: 301-319.

5. Gayda M, Saleh D (2002) Central and peripheral deafness in autistic and deaf children. Ann Méd Psychol 160: 671-676.

6. Lina G, Granade A, Try E (2005) What to do with deafness in the child. Encycl Méd chir Otorhinolaryngology 2: 290-300.

7. Mowat D, Wilson M (2010) Mowat-Wilson syndrome. In :Cassidy SB, Allanson JE (Eds.), Management of genetic syndromes. $3^{\text {rd }}$ Ed. New Jersey, USA.

8. Cacheux V, Dastot Le Moal F, Kaariainen H, Bondurand N, Rintala R, et al. (2001) Loss of function mutations in SIP1 Smad interacting protein 1 result in a syndromic hirschsprung disease. Hum Mol Genet 10(14): 1503-1510.

9. Wakamatsu N, Yamada Y, Yamada K, Ono T, Nomura N, et al. (2001) Mutations in SIP1,encoding Smad interacting protein 1,cause a form of Hirschsprung disease. Nat Genet 27(4): 369-370.

10. Zweier C, Thiel CT, Dufke A, Crow YJ, Meinecke P, et al. (2005) Clinical and mutational spectrum of Mowat-Wilson syndrome. Eur J Med Genet 48(2): 97-111.

11. Paola Cerruti Mainard, Livia GaravellI, Olivier Lyon Caen (2014) Orphonat the portal for rare diseases and orphan drugs.

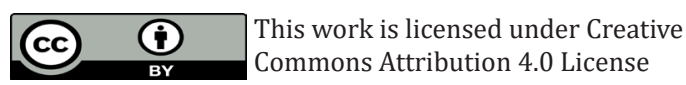

To Submit Your Article Click Here: $\quad$ Submit Article

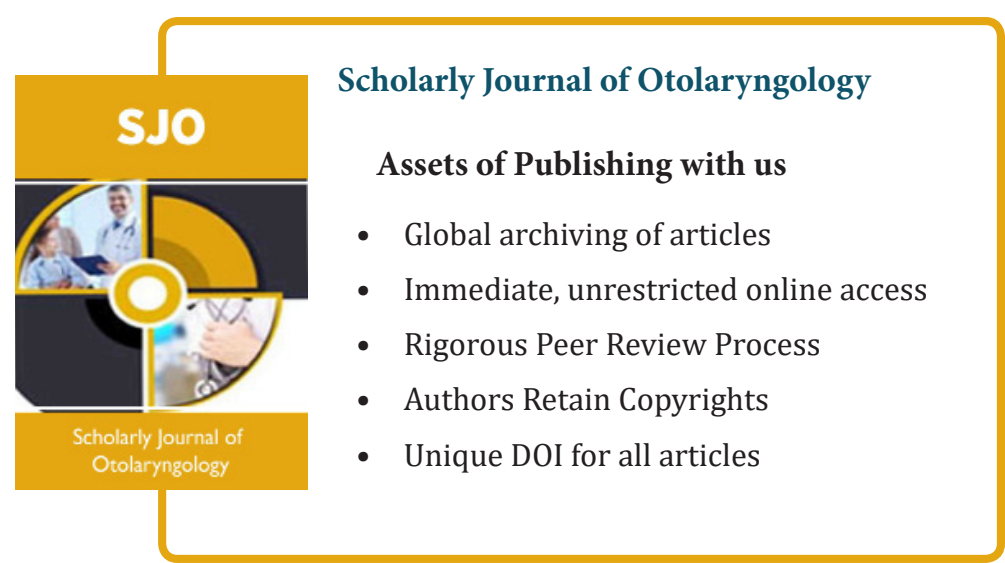

